THU-97/02

hep-th/9701169

\title{
Supersymmetric quantum mechanics, supermembranes and Dirichlet particles $^{\dagger}$
}

\author{
Bernard de Wit \\ Institute for Theoretical Physics \\ Utrecht University \\ Princetonplein 5, 3508 TA Utrecht, The Netherlands
}

\begin{abstract}
We review models of supersymmetric quantum mechanics that are important in the description of supermembranes and of Dirichlet particles, which play a role in the context of M-theory.
\end{abstract}

\footnotetext{
† Invited talk given at the $30^{\text {th }}$ International Symposium Ahrenshoop on the Theory of Elementary Particles, Buckow, August 27 - 31, 1996; to appear in Nuclear Physics B (Proc. Suppl.).
}

January 1997 


\title{
Supersymmetric quantum mechanics, supermembranes and Dirichlet particles
}

\author{
Bernard de $\mathrm{Wit}^{\mathrm{a}}$ \\ ${ }^{a}$ Institute for Theoretical Physics, Utrecht University \\ Princetonplein 5, 3508 TA Utrecht, The Netherlands
}

We review models of supersymmetric quantum mechanics that are important in the description of supermembranes and of Dirichlet particles, which play a role in the context of M-theory.

\section{Supersymmetric quantum mechanics}

Consider the class of supersymmetric Hamiltonians

$$
\begin{aligned}
H= & \frac{1}{2} P_{a}^{A} P_{a A}+\frac{1}{4}\left(f_{A B C} X_{a}^{B} X_{b}^{C}\right)^{2} \\
& -\frac{1}{2} i f_{A B C} \theta^{A} \gamma^{a} X_{a}^{B} \theta^{C},
\end{aligned}
$$

depending on a number of $d$-dimensional coordinates $\vec{X}^{A}(a=1, \ldots, d)$ and corresponding momenta $\vec{P}^{A}$ as well as real spinorial anticommuting coordinates $\theta_{\alpha}^{A}$. The index $A$ labels the vectors and spinors as components of some (compact) Lie algebra $g$ with structure constants $f_{A B C}$. The phase space is restricted to an subspace invariant under the corresponding Lie group $G$, and is therefore subject to the constraints

$\varphi_{A}=f_{A B C}\left(\vec{X}^{B} \cdot \vec{P}^{C}-\frac{1}{2} i \theta^{B} \theta^{C}\right) \approx 0$.

The above models were proposed long ago as extended models of supersymmetric quantum mechanics with more than four supersymmetries [1]. The spatial dimension $d$ and the corresponding spinor dimension is severely restricted. The models exist for $d=2,3,5$, or 9 dimensions; the (real) spinor dimension equals $2,4,8$, or 16 , respectively. Naturally this is also the number of independent supercharges, defined by

$Q=\left(P_{a}^{A} \gamma^{a}+\frac{1}{2} f_{A B C} X_{a}^{B} X_{b}^{C} \gamma^{a b}\right) \theta^{A}$.

These charges generate the familiar supersymmetry algebra (in the group-invariant subspace),

$\left\{Q_{\alpha}, Q_{\beta}\right\} \approx 2 H \delta_{\alpha \beta}$.
At the time these models were proposed it was recognized that they coincide precisely with the zero-volume limit of supersymmetric gauge theories. Hence one suppresses the spatial dependence of the vector and spinor fields, while the time-like component of the gauge field is set to zero by a gauge choice:

$A_{\mu}^{A}(\mathrm{x}, t) \rightarrow\left\{\begin{array}{l}A_{a}^{A}(t), \\ A_{0}^{A}=0,\end{array} \quad \theta^{A}(\mathrm{x}, t) \rightarrow \theta^{A}(t)\right.$.

The constraint equations (2) then correspond to Gauss' law. These Yang-Mills theories are formulated in Minkowski spacetimes of dimension 3, 4, 6 , or 10 .

Let us briefly mention a number of characteristic features that are extremely relevant for what follows. We note the appearance of "valleys" where the potential in (11) vanishes. This happens whenever the coordinates $\vec{X}^{A}$ are restricted to take values in an abelian subalgebra. These valleys extend all the way to infinity where they become increasingly narrow. Their existence raises questions about the nature of the spectrum of (11). In the bosonic versions of these models the wave function cannot freely extend to infinity, because at large distances it becomes more and more squeezed in the valley. By the uncertainty principle, this gives rise to kinetic-energy contributions which increase monotonically along the valley. Another way to see this is by noting that the oscillations perpendicular to the valleys give rise to a zero-point energy, which induces an effective potential barrier that confines the wave function. This confinement causes the spectrum 
to be discrete [2]. However, for the supersymmetric models defined by (11) the situation is different. Supersymmetry can cause a cancelation of the transverse zero-point energy. Then the wave function is no longer confined, indicating that the supersymmetric models have a continuous spectrum. The latter was proven in [3].

At the moment there is no proof that the Hamiltonian (11) allows normalizable or localizable zero-energy states, superimposed on the continuous spectrum. The relevance of such states will be discussed later on. There is an important difference between states whose energy is exactly equal to zero and states of positive energy. Because of the supersymmetry algebra, zero-energy states must be annihilated by the supercharges. Hence, they are supersinglets. These singlets may have some degeneracy, for instance, associated with some other symmetries such as rotational invariance. The positive-energy states, on the other hand, must constitute full supermultiplets. So they are multiplets consisting of multiples of $1+1,2+2,8+8$, or $128+128$ bosonic + fermionic states, corresponding to $d=2,3,5$ or 9 , respectively.

\section{Supermembranes}

Fundamental supermembranes can be described in terms of actions of the Green-Schwarz type, possibly in a nontrivial (super)spacetime background [4, 5]. Such actions exist for supersymmetric $p$-branes, where $p=0,1, \ldots$ defines the spatial dimension of the brane. Thus for $p=0$ we have a superparticle, for $p=1 \mathrm{a}$ superstring, for $p=2$ a supermembrane, and so on. The dimension of spacetime in which the superbranes can live is very restricted [6]. These restrictions arise from the fact that the action contains a Wess-Zumino-Witten term, whose supersymmetry depends sensitively on the spacetime dimension. If the coefficient of this term takes a particular value then the action has an additional fermionic gauge symmetry, the so-called $\kappa$ symmetry. This symmetry is necessary to ensure the matching of (physical) bosonic and fermionic degrees of freedom. In the following we restrict ourselves to supermembranes (i.e., $p=2$ ) in eleven dimensions.

In the notation and conventions of [7] the supermembrane Lagrangian reads

$$
\begin{aligned}
& \mathcal{L}=-\sqrt{-g(X, \theta)}-\epsilon^{i j k} \bar{\theta} \Gamma_{\mu \nu} \partial_{k} \theta \\
& \times\left[\frac{1}{2} \partial_{i} X^{\mu}\left(\partial_{j} X^{\nu}+\bar{\theta} \Gamma^{\nu} \partial_{j} \theta\right)+\frac{1}{6} \bar{\theta} \Gamma^{\mu} \partial_{i} \theta \bar{\theta} \Gamma^{\nu} \partial_{j} \theta\right],
\end{aligned}
$$

where $X^{\mu}(\zeta)$ and $\theta(\zeta)$ denote the superspace coordinates of the membrane parametrized in terms of world-volume parameters $\zeta^{i}(i=0,1,2)$. The fermionic coordinates $\theta$ are 32-component Majorana spinors. The gamma matrices are denoted by $\Gamma^{\mu}$; gamma matrices with more than one index denote antisymmetrized products of gamma matrices in the usual fashion. The metric $g_{i j}(X, \theta)$ is the induced metric on the world volume,

$g_{i j}=\left(\partial_{i} X^{\mu}+\bar{\theta} \Gamma^{\mu} \partial_{i} \theta\right)\left(\partial_{j} X^{\nu}+\bar{\theta} \Gamma^{\nu} \partial_{j} \theta\right) \eta_{\mu \nu}$,

where $\eta_{\mu \nu}$ is the eleven-dimensional Minkowski metric. It is easy to see that $g_{i j}$, and therefore the first term in (7), is invariant under spacetime supersymmetry. In $4,5,7$, or 11 spacetime dimensions the second term proportional to $\epsilon^{i j k}$ is also supersymmetric (up to a total divergence) and the full action is invariant under $\kappa$-symmetry.

In order to study the quantum-mechanical properties of the supermembrane we use the lightcone gauge. First we define light-cone coordinates

$$
X^{\mu}=\left\{\begin{array}{l}
X^{ \pm}=\frac{1}{\sqrt{2}}\left(X^{10} \pm X^{0}\right) \\
X^{a} \quad(a=1, \ldots, 9)
\end{array}\right.
$$

with a similar decomposition of the gamma matrices $\Gamma^{\mu}$ into $\gamma^{ \pm}$and $\gamma^{a}(a=1, \ldots, 9)$. It is possible to impose the gauge conditions 1710

$$
\begin{aligned}
X^{+}(\zeta) & =X^{+}(0)+\tau, \\
\partial_{r} X^{-} & =-\frac{1}{P^{+}}\left(\vec{P} \cdot \partial_{r} \vec{X}+P^{+} \bar{\theta} \gamma_{-} \partial_{r} \theta\right), \\
P^{+} & =P_{0}^{+} \sqrt{w(\sigma)}, \quad \gamma_{+} \theta=0,
\end{aligned}
$$

where $\tau=\zeta^{0}$ and the remaining two parameters $\sigma^{r}=\left(\zeta^{1}, \zeta^{2}\right)$ parametrize the spatial extension of the membrane. We have introduced conjugate momenta and a density $\sqrt{w(\sigma)}$ normalized according to $\int \mathrm{d}^{2} \sigma \sqrt{w(\sigma)}=1$. Therefore the constant $P_{0}^{+}$represents the membrane centre-of-mass 
$(\mathrm{CM})$ momentum in the direction associated with the coordinate $X^{-}$,

$P_{0}^{+}=\int \mathrm{d}^{2} \sigma P^{+}$.

The other CM coordinates and momenta are

$\vec{P}_{0}=\int \mathrm{d}^{2} \sigma \vec{P}, \quad \vec{X}_{0}=\int \mathrm{d}^{2} \sigma \sqrt{w(\sigma)} X(\sigma)$,

$\theta_{0}=\int \mathrm{d}^{2} \sigma \sqrt{w(\sigma)} \theta(\sigma)$.

In the light-cone gauge we are left with the transverse coordinates $\vec{X}$ and corresponding momenta $\vec{P}$, which transform as vectors under the $S O(9)$ group of transverse rotations. Only sixteen fermionic components $\theta$ remain, which transform as $S O(9)$ spinors. Furthermore we have the $\mathrm{CM}$ momentum $P_{0}^{+}$and the CM coordinate $X_{0}^{-}$(the remaining modes in $X^{-}$are dependent), while the CM momentum $P_{0}^{-}$is equal to minus the supermembrane Hamiltonian.

The supermembrane Hamiltonian takes the following form

$H=\frac{\vec{P}_{0}^{2}}{2 P_{0}^{+}}+\frac{\mathcal{M}^{2}}{2 P_{0}^{+}}$,

where $\mathcal{M}$ is the supermembrane mass operator, which does not depend on any of the CM coordinates or momenta. After some changes in notation of the spinors, the expression for $\mathcal{M}^{2}$ is

$$
\begin{aligned}
& \mathcal{M}^{2}=\int \mathrm{d}^{2} \sigma \sqrt{w(\sigma)} \\
& \times\left[\frac{\left[\vec{P}^{2}(\sigma)\right]^{\prime}}{w(\sigma)}+\frac{1}{2}\left(\left\{X^{a}, X^{b}\right\}\right)^{2}-i \theta \gamma_{a}\left\{\theta, X^{a}\right\}\right],
\end{aligned}
$$

where $\left[\vec{P}^{2}\right]^{\prime}$ indicates that the contribution of the CM momentum $\vec{P}_{0}$ is suppressed. We made use of a Lie bracket $\{A, B\}$, which, for two functions $A(\sigma)$ and $B(\sigma)$, is defined by

$$
\{A, B\}(\sigma) \equiv \frac{1}{\sqrt{w(\sigma)}} \epsilon^{r s} \partial_{r} A(\sigma) \partial_{s} B(\sigma) .
$$

It is straightforward to show that (13) satisfies the Jacobi identity. We stress that we assume that the membrane embedding coordinates are single-valued functions so that the contributions of the $\mathrm{CM}$ coordinates cancel also in the second and third term under the integral in (12).

The mass operator is accompanied by a constraint, which follows from the integrability of the second gauge condition (8) for $\partial_{r} X^{-}$,

$\varphi=\left\{X^{a}, P^{a}\right\}-\frac{1}{2} i\left\{\theta_{\alpha}, \theta_{\alpha}\right\} \approx 0$.

Apart from its $\mathrm{CM}$ value, $X^{-}$can then be determined in terms of the other coordinates and momenta.

The Hamiltonian commutes with 32 supercharges. Half of them are proportional to the fermionic $\mathrm{CM}$ spinor coordinates $\theta_{0}$. Therefore they have no bearing on the mass operator. The other 16 charges take the form

$Q=\int \mathrm{d}^{2} \sigma\left[P^{a} \gamma_{a}+\frac{1}{2} \sqrt{w}\left\{X^{a}, X^{b}\right\} \gamma_{a b}\right] \theta$.

These charges involve both the CM momenta $\vec{P}_{0}$ and the modes contained in the mass operator (12). They satisfy the supersymmetry algebra, in the constrained subspace,

$\left\{Q_{\alpha}, Q_{\beta}\right\} \approx \delta_{\alpha \beta} \mathcal{M}^{2}$,

The structure of the Hamiltonian (11) shows that the wave functions for the supermembrane now factorize into a wave function pertaining to the CM modes and a wave function of the supersymmetric quantum-mechanical system that describes the other modes. For the latter the mass operator plays the role of the Hamiltonian. When the mass operator vanishes on the state, then the 32 supercharges act exclusively on the CM coordinates and generate a massless supermultiplet of eleven-dimensional supersymmetry. In case there is no other degeneracy beyond that caused by supersymmetry, the resulting supermultiplet is the one of supergravity, describing the graviton, the antisymmetric tensor and the gravitino. In terms of the $S O(9)$ helicity representations, it consists of $\mathbf{4 4} \oplus \mathbf{8 4}$ bosonic and $\mathbf{1 2 8}$ fermionic states. When the mass operator does not vanish on the states, we are dealing with huge supermultiplets consisting of multiples of $2^{15}+2^{15}$ states. 


\section{Supermembrane as models in supersym- metric quantum mechanics}

The gauge conditions (8) adopted above leave a residual invariance consisting of so-called areapreserving diffeomorphisms. They are defined by $\sigma^{r} \rightarrow \sigma^{r}+\xi^{r}(\sigma)$ with

$\partial_{r}\left(\sqrt{w(\sigma)} \xi^{r}(\sigma)\right)=0$.

The general solution of (17) can be decomposed into co-exact and harmonic vector fields. For a membrane of genus $g$ there are precisely $2 g$ independent harmonic vectors. Furthermore, there can be homotopically nontrivial transformations; these will not be considered in what follows. The co-exact components are parametrized in terms of globally defined functions $\xi(\sigma)$,

$\xi^{r}(\sigma)=\frac{\epsilon^{r s}}{\sqrt{w(\sigma)}} \partial_{s} \xi(\sigma)$,

and generate an invariant subgroup, which we denote by $G$. In the following, we restrict our attention to this invariant subgroup when refering to area-preserving transformations. The commutator of two infinitesimal $G$-transformations characterized by functions $\xi_{1}$ and $\xi_{2}$ yields a third $G$ transformation characterized by $\xi_{3}$, which equals

$\xi_{3}=\left\{\xi_{2}, \xi_{1}\right\}$

According to (19) the bracket (13) thus encodes the structure constants of the group $G$. This interpretation shows that the supermembrane mass operator (12) belongs to the class of supersymmetric Hamiltonians (11). The constraint equation (14) restricts the physical states to be invariant under the area-preserving diffeomorphisms. Clearly the supermembrane in a $(d+2)$-dimensional spacetime can be regarded as a limiting case of the supersymmetric quantum mechanical models in $d$ dimensions discussed in section 1 .

In order to elucidate this relation one expands all coordinates and momenta into a complete orthonormal basis of functions consisting of the constant function 1 and functions $Y^{A}(\sigma)$ (where $A=1,2, \ldots, \infty)$. The coefficient of 1 represents the $\mathrm{CM}$ value, so that we write

$\vec{X}(\sigma)=\vec{X}_{0}+\sum_{A=1}^{\infty} \vec{X}_{A} Y^{A}(\sigma)$,

with similar expansions for the other coordinates and momenta. The basis functions $Y^{A}$, which can be chosen real or complex, are normalized according to

$\int \mathrm{d}^{2} \sigma \sqrt{w(\sigma)} Y^{A}(\sigma)=0$,

$\int \mathrm{d}^{2} \sigma \sqrt{w(\sigma)} Y^{A}(\sigma) Y_{B}(\sigma)=\delta_{B}^{A}$,

where indices $A, B$ are raised and lowered by complex conjugation. Their completeness implies

$\sum_{A=1}^{\infty} Y^{A}(\sigma) Y_{A}\left(\sigma^{\prime}\right)=\frac{1}{\sqrt{w(\sigma)}} \delta^{(2)}\left(\sigma, \sigma^{\prime}\right)-1$.

The bracket $\left\{Y^{A}, Y^{B}\right\}$ can be expressed in terms of the $Y^{A}$ and one derives

$\left\{Y^{A}, Y^{B}\right\}=f^{A B}{ }_{C} Y^{C}$,

so that the constants $f{ }_{C}^{A B}$ represent the structure constants of the infinite-dimensional group $G$. Other tensors related to the diffeomorphisms generated by harmonic vectors and tensors needed for the Lorentz algebra generators were defined in 111].

If we replace $G$ by a finite group, then (13) defines the Hamiltonian of a supersymmetric quantum-mechanical system. In the limit to the infinite-dimensional group $G$ we thus recover the supermembrane. This observation enables one to regularize the supermembrane in a supersymmetric way by considering a limiting procedure based on a sequence of groups whose limit yields the group $G$. For closed membranes of certain topology it is known how to approximate the group $G$ as a particular $N \rightarrow \infty$ limit of $S U(N)$. To be precise, it can be shown that the structure constants of $S U(N)$ tends to those of $G$ up to corrections of order $1 / N^{2}$. The nature of this limit is subtle and depends on the membrane topology. As long as $N$ is finite, no distinction can be made with regard to the topology. In some sense, all topologies are thus included at the level of finite $N$. Another subtlety concerns the emergence 
of diffeomorphisms associated with the harmonic vectors, which cannot be incorporated for finite $N$, at least not in infinitesimal form. For a discussion of these subtleties we refer to [11,12].

The classical configurations of zero energy are now characterized by $\left\{X_{a}, X_{b}\right\}=0$. This implies that the membrane collapses into stringlike configurations of zero area. The same feature exists for general $p$-branes. Classical (super)p-branes are unstable: the zero-energy configurations correspond to collapsed branes of lower dimensionality $p-1$. Whether or not this unstability persists at the quantum level is the subject of the next section. Of all $p$-branes, only the (super)particle and the (super)string do not suffer from the above instability. The particle simply because it has no internal structure at all, and the string because, apart from its centre-of-mass motion, all modes are confined by harmonic-oscillator potentials.

\section{Continuous spectrum}

As we pointed out above supermembranes and the supersymmetric quantum-mechanical models introduced in section 1 share the important feature that the potential vanishes in certain valleys that extend to infinity. In the presence of these valleys, there is a latent danger that the wave functions will no longer be confined. In that case there is then no obvious reason why the spectrum should be discrete. However, as already discussed in section 1, quantum-mechanical effects may still prevent the wave function from escaping through the zero-energy valley. To make this more explicit, consider the following twodimensional Hamiltonian,

$H_{B}=p_{x}^{2}+p_{y}^{2}+x^{2} y^{2}$.

Obviously the potential in 24 has zero-energy valleys along the $x$ - and the $y$-axis. Nevertheless the eigenfunctions of (24) are confined and cannot escape through these valleys. This follows from decomposing 242) as

$H_{B}=\frac{1}{2}\left(p_{x}^{2}+p_{y}^{2}\right)+H_{1}+H_{2}$,

where $H_{1}=\frac{1}{2} p_{x}^{2}+\frac{1}{2} x^{2} y^{2}$ and $H_{2}=\frac{1}{2} p_{y}^{2}+\frac{1}{2} x^{2} y^{2}$. Since $H_{1}$ and $H_{2}$ take the form of harmonic oscillator Hamiltonians in $x$ and $y$, respectively, with frequencies equal to $|x|$ or $|y|$, we immediately derive the operator inequality $(\hbar=1)$

$\left.H_{B} \geq \frac{1}{2}\left(p_{x}^{2}+p_{y}^{2}\right)\right)+\frac{1}{2}(|x|+|y|)$.

The operator on the right-hand side has a discrete spectrum as the wave function will be confined by the infinitely rising potential. Therefore the spectrum of $H_{B}$ is discrete [2].

It is now obvious why the introduction of supersymmetry could drastically change the situation described above. As is well-known, supersymmetric harmonic oscillators have no zero-point energy, so that the confining effective potential may vanish. Whether or not the potential in the valley will vanish completely can be investigated in a simple two-dimensional model [3]. We first introduce a supersymmetry charge by

$Q=Q^{\dagger}=\sigma_{x} p_{x}+\sigma_{y} p_{y}+\sigma_{z} x y$,

where $\sigma_{x}, \sigma_{y}, \sigma_{z}$ are the Pauli spin matrices, so that $Q$ acts in a two-dimensional fermionic space. The Hamiltonian then follows in the usual fashion,

$H=Q^{2}=\left(\begin{array}{cc}H_{B} & x+i y \\ x-i y & H_{B}\end{array}\right)$.

In order to establish the absence of an effective potential barrier that may prevent the wave function from escaping through the valley, we consider a set of normalized trial wave functions

$\psi_{\lambda}(x, y)=\chi(x-\lambda) \varphi_{0}(x, y) \xi_{\mathrm{F}}$,

characterized by some parameter $\lambda$. Here $\chi$ is a one-dimensional free-particle wave packet satisfying $\int \mathrm{d} x|\chi|^{2}=1$, which has compact support so that (29) is only different from zero for $x \approx \lambda, \varphi_{0}$ is the normalized groundstate wave function for a one-dimensional harmonic oscillator,

$\varphi_{0}(x, y)=\pi^{-\frac{1}{4}}|x|^{\frac{1}{4}} \exp \left(-\frac{1}{2}|x| y^{2}\right)$,

and $\xi_{\mathrm{F}}$ is a normalized two-dimensional spinor. When the parameter $|\lambda|$ is large, the wave function (29) thus has its support in a narrow region along the $x$-axis. Irrespective of the precise form of $\chi(x-\lambda)$, we have the identity

$Q \psi_{\lambda}=\left(\sigma_{x} p_{x}+x y \sigma_{z}\left(\operatorname{sgn} x \sigma_{x}+\mathbf{1}\right)\right) \psi_{\lambda}$. 
By suitably choosing the spinor $\xi_{\mathrm{F}}$ the second term vanishes, so that

$Q \psi_{\lambda}=\sigma_{x} p_{x} \psi_{\lambda}=-\operatorname{sgn} x p_{x} \psi_{\lambda}$.

One can easily verify that for this choice of $\xi_{\mathrm{F}}$ the zero-point energy in $H_{B}$ associated with the transverse oscillations (i.e., in $y$ ) cancels against the fermionic zero-point energy induced by the term $x \sigma_{x}$ in the Hamiltonian (28).

What we now intend to show is that, by making $|\lambda|$ large, thus pushing the domain of support of the wave function deeper into the valley, $\psi_{\lambda}$ will tend to a supersymmetric wave function, which, by virtue of the supersymmetry algebra, must have zero energy. To see how this works, we derive the following result for any finite power $\nu$ of $Q$ acting on $\psi_{\lambda}$,

$$
\left\|Q^{\nu} \psi_{\lambda}\right\|^{2}=\left|\left(p_{x}\right)^{\nu} \chi\right|^{2}+\mathrm{O}\left(\lambda^{-1}\right),
$$

where the first term on the right-hand side is obvious. It represents the norm of the onedimensional wave function $\left(p_{x}\right)^{\nu} \chi$, which is equal to the $\nu$-th power of the energy of the wave packet since $\chi$ is normalized to unity. The second term originates from operators $p_{x}$ acting on $\varphi_{0}$. This introduces factors $|x|^{-1}$ or $y^{2}$; the latter become also proportional to $|x|^{-1}$ upon integrating over $y$. As $x \approx \lambda$, the contributions from $p_{x} \varphi_{0}$ are thus of order $\lambda^{-1}$.

Obviously the right-hand side of (33) can be made arbitrarily small by choosing a wave packet $\chi$ of sufficiently low energy and by making $\lambda$ sufficiently large. The latter implies that the wave function will extend further and further into the valley, so that there is apparently no confining force, as $\psi_{\lambda}$ approaches a supersymmetric wave function with zero energy. Furthermore we derive along the same lines, for any $E$,

$$
\left\|(H-E) \psi_{\lambda}\right\|^{2}=\left|\left(p_{x}^{2}-E\right) \chi\right|^{2}+\mathrm{O}\left(\lambda^{-1}\right) .
$$

For any positive $E$ and $\varepsilon$ we can then choose a wave packet $\chi$ such that

$\left|\left(p_{x}^{2}-E\right) \chi\right|^{2} \leq \varepsilon / 2$.

By making $\lambda$ sufficiently large we can make the $\mathrm{O}\left(\lambda^{-1}\right)$ corrections in (34) smaller than $\varepsilon / 2$.
Combining (34) and (35) then shows that, with $\left\|\psi_{\lambda}\right\|=1$,

$$
\left\|(H-E) \psi_{\lambda}\right\|^{2} \leq \varepsilon
$$

for any positive $E$ and $\varepsilon$. This proves that any nonnegative $E$ is a spectral value of the Hamiltonian $H$, so that the spectrum is continuous. It is thus clear that the reason for the continuity of the spectrum is that wave functions can escape to infinity along the valleys that have zero classical energy.

The above example exhibits the same qualitative features as the supersymmetric quantummechanical models of section 1 . Using the existence of the potential valleys extending to infinity and supersymmetry, it has been proven that also the Hamiltonian (1) has a continuous spectrum starting at zero for any finite (compact) gauge group [3]. By contrast the spectrum of the corresponding bosonic theory is discrete [2]. The mass operator of the supermembrane can be regarded as the $N \rightarrow \infty$ limit of the supersymmetric Hamiltonian (11) for the group $S U(N)$. Therefore, modulo unexpected subtleties associated with this limit, the supermembrane mass spectrum is also continuous.

\section{Discrete states}

The result reported above indicates that the exact supermembrane is not a viable model for elementary particles. Still there remains the issue of finding discrete and possibly zero-energy eigenstates. Discrete eigenstates within the continuum are in principle possible, although such examples are not easy to construct and always suffer from instabilities (as the discrete states can "decay" into the nearby continuum). The problem is somewhat simpler to address for the zeroenergy states, where it boils down to the question of whether there exist normalizable solutions of $Q \psi=0$.

One might attempt to determine the nature of the ground state from the value of Witten index [13], which does not require an explicit derivation of the ground-state wave functions. As is well known, if this index is nonzero there must be an unequal number of zero-energy fermionic and 
bosonic states. At least some of them must be annihilated by the supersymmetry charge. Motivated by the connection of the supersymmetric quantum mechanics with supersymmetric YangMills theories, the Witten index has been determined for $G=S U(2)$ in the so-called ultralocal limit 14]. Usually one finds that the index is different from zero, with the exception of the two-dimensional models (corresponding to a supermembrane moving in a four-dimensional space-time), where the index vanishes for odddimensional gauge groups $G$. In 15] a "twisted" version of the Witten index was proposed which was argued to be strictly positive. However, it is known that, for a continuous spectrum without a gap, the very notion of the Witten index will sensitively depend on the regularization that one employs [16. In any case, the issue cannot just be resolved by formal manipulations of certain functional integrals. Rather than being positive the result is likely to be ill-defined!

Early attempts to determine directly whether or not the theory exhibits zero-energy ground states were described in [7]. Admittedly, the results of this work remained somewhat inconclusive, but all the evidence seemed to point in the direction of no normalizable zero-energy states. Nevertheless, even for the simple two-dimensional model of the previous section, it is not known whether or not normalizable zero-energy states exist [18].

To illustrate some of the subtleties that one may encounter when analyzing this question, let us momentarily return to a variant of this twodimensional model, where the supercharge depends on some superpotential $W(x, y)$,

$Q=\sigma_{x} p_{x}+\sigma_{y} p_{y}+\sigma_{z} W(x, y)$.

Possible valleys are associated with orbits consisting of points $(x, y)$ for which $W(x, y)$ vanishes. The presence of an arbitrary function $W$ offers a welcome feature, as it allows the study of the degenerate potentials as a limiting case of potentials that are better behaved and are therefore more amenable to rigorous analysis. However, here we simply restrict ourselves to the case that $W$ is rotationally invariant, so that it depends only on the radius $r$. This means that possible valleys take the form of circles in the $x-y$ plane. After passing to spherical coordinates, $r$ and $\varphi$, one finds that the following two wave functions are annihilated by the supercharge (37),

$$
\begin{aligned}
\psi_{ \pm}(r, \varphi)=\frac{1}{\sqrt{r}} & \exp \left[\mp \int_{r_{0}}^{r} \mathrm{~d} r^{\prime} W\left(r^{\prime}\right)\right] \\
\times & \left(\begin{array}{c}
\mathrm{e}^{-i \varphi / 2} \\
\mathrm{e}^{i(\varphi \pm \pi) / 2}
\end{array}\right) .
\end{aligned}
$$

Depending on the behaviour of $W(r)$ at large $r$, one of these solutions can be square-integrable. A conspicuous feature is their behaviour at small distances. If $W(r)$ is regular near the origin, then the wave function exhibits a $1 / \sqrt{r}$ singularity. One may wonder whether such a singularity, while leaving the wave function square-integrable, is acceptable. With such a singularity the kineticenergy term in the Hamiltonian is not positive definite, although for (38) the kinetic energy still cancels against the potential energy. Extra care is required when integrating by parts, where one may pick up boundary contributions at $r=0$.

To analyze the situation in a little more detail, let us decompose the Hilbert space in eigenspaces of the angular-momentum operator. The corresponding wave functions can be written as

$\psi(r, \varphi)=\left(\begin{array}{c}\mathrm{e}^{-i s \varphi} f_{1}(r) \\ \mathrm{e}^{-i(s-1) \varphi} f_{2}(r)\end{array}\right)$,

where we expect that the relevant spin-values are given by $s=\frac{1}{2}(1+n)$ with $n$ an integer. One easily establishes that the supercharge (37) acts in subspaces of given spin. In each of these subspaces one can verify that the supercharge is selfadjoint (which is crucial for proving that the spectrum of the Hamiltonian is nonnegative!), provided the following condition is satisfied,

$\lim _{r \downarrow 0} r\left(\phi, \sigma_{x} \psi\right)=0$.

Here $\phi(r, \varphi)$ and $\psi(r, \varphi)$ are two two-component wave functions of the same spin (so that the expression in (40) is $\varphi$-independent) and $($, , denotes the complex inner product for two-spinors. It turns out that precisely one of the wave functions (38) can satisfy the above condition. Of 
course, this is no problem, as we already know that only one of them can be square-integrable. The same result is obtained when evaluating the condition required for the self-adjointness of the Hamiltonian. It reads

$\lim _{r \downarrow 0} r\left[\left(\phi, \partial_{r} \psi\right)-\left(\partial_{r} \phi, \psi\right)\right]=0$.

Again we have to exclude one of the wave functions (38) in order that the condition be satisfied.

Hence we may conclude that certain mild singularities are acceptable in the wave function. This, however, has consequences for other potential eigenfunctions, so that a proper analysis is no longer restricted to a single wave function. Obviously, the presence of the singularities forms an extra complication in obtaining rigorous results for zero-energy states.

Establishing the existence or nonexistence of zero-energy states for the supersymmetric models of section 1 with finite-dimensional groups, is even more difficult. The decomposition of the wave function in terms of the anticommuting coordinates gives rise to a multicomponent wave function. For the smallest case, namely that of the group $S U(2)$ in $d=2$ dimensions, the group-invariant states are characterized in terms of three-component wave functions. This theory was analyzed in [7], where various arguments against the existence of zero-energy states were presented. Recently, a rigorous proof was presented that, indeed, normalizable zero-energy states do not exist for $S U(2)$ and $d=2$ [17.

For $d=3$ there are also indications that no normalizable zero-energy states exist. Here we have coordinates $\vec{X}^{A}$ and complex two-component spinors $\theta^{A}$. The supercharges can be written as

$Q_{\alpha}=\frac{1}{i} \mathrm{e}^{W} \frac{\partial}{\partial \vec{X}^{A}} \mathrm{e}^{-W}\left(\vec{\sigma} \theta^{A}\right)_{\alpha}$,

$Q_{a}^{\dagger}=\frac{1}{i} \mathrm{e}^{-W} \frac{\partial}{\partial \vec{X}^{A}} \mathrm{e}^{W}\left(\frac{\partial}{\partial \theta^{A}} \vec{\sigma}\right)_{\alpha}$,

and the superpotential $W$ reads

$W=\frac{1}{6} f_{A B C} \varepsilon^{a b c} X_{a}^{A} X_{b}^{B} X_{c}^{C}$.

Clearly $\exp [ \pm W]$ is not normalizable as $W$ is odd in the coordinates. This directly precludes the existence of normalizable zero-energy states with zero or maximal fermion number. To determine whether the same result holds for states of intermediate fermion-number values, is not so easy, although it seems likely that the exponential factor will always cause a certain lack of normalizability. Unfortunately, more rigorous results regarding this question are still lacking.

For other groups and in higher dimensions, an explicit evaluation of the resulting system of equations becomes untractable, because the number of components of the wave function increases exponentially with the spinor dimension times the group dimension.

\section{Interpretation of the continuum}

In the context of the elementary supermembrane, the continuity of the spectrum is clearly an undesirable result [12]. However, one may turn things around and wonder whether there exists a context in which a continuous spectrum forms a more welcome feature. Obviously, such a spectrum would arise naturally when considering a many-particle Hilbert space of states associated with well-separated almost-free particles. Of course, the situation is rather subtle, as there should be no energy gap. The continuous spectrum starts at zero and possible bound states must be superimposed on this continuum.

There are objects that arise in the context of more complicated theories based on an infinite number of degrees of freedom, whose spectrum exhibits some of the characteristic features discussed above. The interesting point to note is that certain nontrivial properties of this spectrum may be reflected in the spectrum of a corresponding quantum-mechanical model. In this way these properties can thus be explored in the context of the conceptually more simple quantummechanical models based on a finite number of degrees of freedom.

The above strategy has been applied successfully to the case of BPS monopoles and dyons of four-dimensional $N=4$ supersymmetric gauge theories. Static multi-monopole states of given energy and electric charge are contained in a multi-parameter family of solutions. The parameters that label these degenerate solutions are the 
collective coordinates, or moduli, and the space parametrized by these coordinates is called the moduli space. For multi-monopole solutions the collective coordinates are associated with the locations of the monopoles and their electric charge. In the supersymmetric case there will also be fermionic moduli. A detailed discussion of this is outside the scope of this lecture and we refer the reader to a recent set of lecture notes by Harvey 19. The multi-monopole configurations can exist as static solutions, because the force between the static monopoles cancels. The repulsive Coulomb force cancels against the force mediated by the exchange of scalar particles. This cancelation can be understood from the fact that the Bogomol'nyi bound is saturated. It is known that the moduli space of BPS multi-monopole configurations must be a hyper-Kähler space [20].

One may also consider configurations of slowly moving monopoles by allowing the collective coordinates to depend on time. The dynamics of the multi-monopole solutions can then be described in terms of a relatively simple quantummechanical model defined on a $4 n$-dimensional hyper-Kähler space, where $n$ denotes the number of monopoles. In $N=4$ Yang-Mills theory the BPS monopoles break half of the number of supersymmetries, so that the moduli space is extended with $8 n$ fermionic collective coordinates, which are grouped into $4 n$ real spinor doublets related by supersymmetry to the $4 n$ bosonic moduli. After extracting the centre-of-mass collective coordinates corresponding to position and total charge, one is thus left with supersymmetric quantum mechanics based on a $(4 n-4)$ dimensional Kähler space [21]. The model has four independent supersymmetries parametrized by real anticomuting doublet parameters, which are related to the eight supersymmetries that were left invariant in the underlying field theory by the BPS configurations.

From a conjectured property of the fourdimensional gauge theory, namely $S$ duality, one deduces that there should exist a bound state that satifies the Bogomol'nyi bound with electric charge $q$ and magnetic charge $n$, for each $q$ and $n$ relatively prime. This implies that the corresponding $(4 n-4)$-dimensional quantum mechan- ics model must have a supersymmetric ground state. For this type of models a supersymmetric state requires the existence of a certain harmonic form on the hyper-Kähler space [22]. The above arguments were presented by Sen [23], who then demonstrated, using existing results for the twomonopole moduli space [24, that a corresponding harmonic form of the required characteristics does exist. A more general proof for an arbitrary number of monopoles was presented in [25].

Hence from a study of supersymmetric systems based on a finite number of degrees of freedom, one can verify the implications of a nonperturbative field-theoretic phenomenon, such as $S$ duality. However, we should point out that the models that are relevant for the multi-monopole states belong to a different class than the models of section 1. Unlike the latter they are not defined in terms of a Lie group and neither are they subject to a Gauss constraint. They can be obtained upon dimensional reduction from supersymmetric sigma models and not from supersymmetric gauge theories.

The models of section 1 have another role to play. In string theory strings can end on certain defects by means of Dirichlet boundary conditions. These defects are therefore called D-branes (for further references, see [26]). They can have a certain $p$-dimensional spatial extension, and carry Ramond-Ramond charges [27]. These D-branes play an important role in the nonperturbative behaviour of string theory. Here we concentrate on the D0-branes (or Dirichlet particles).

It is not so difficult to determine the effective short-distance description for D-branes [28]. As the strings must be attached to the $p$-dimensional branes, we are dealing with open strings whose endpoints are attached to a $p$-dimensional subspace. At short distances, the interactions caused by these open strings are determined by the massless states of the open string, which constitute the ten-dimensional Yang-Mills supermultiplet, propagating in a $(p+1)$-dimensional spacetime. Because the endpoints of open strings carry Chan-Paton factors the effective short-distance behaviour of $n$ D-branes is described in terms of an $U(n)$ ten-dimensional supersymmetric gauge theory reduced to the $(p+1)$-dimensional world 
volume of the D-brane. The $U(1)$ subgroup describes the centre-of-mass motion of the $n \mathrm{D}$ branes.

In the type-IIA superstring one is dealing with Dirichlet particles moving in nine dimensions. As the world volume is one-dimensional $(p=0)$, the short-distance interactions between these particle is thus described by the model of section 1 with gauge group $U(n)$ and $d=9$. The continuous spectrum without gap is natural here, as it is known that, for static D-branes, the Ramond-Ramond repulsion cancels against the gravitational and dilaton atraction, a similar phenomenon as for BPS monopoles [27]. With this gauge group the coordinates can be described in terms of $n \times n$ hermitean matrices. The valley configurations correspond to the situation where these matrices can be diagonalized simultanously. Hence the coordinates of the model are restricted to nine diagonal matrices whose eigenvalues define the positions of $n$ D-particles. As soon as one or several of these particles coincide then the $[U(1)]^{n}$ symmetry that is left invariant in the valley, will be enhanced to a nonabelian subgroup of $U(n)$. Clearly the degrees of freedom of the model are more than just the D-particle coordinates. The additional degrees of freedom correspond to the strings stretching between the D-particles. Note also that the model naturally incorporates configurations corresponding to widely separated clusters of D-particles, each of which can be described by a supersymmetric quantum mechanics model based on $U(k)$ subgroups of $U(n)$. When all the D-particles move further apart this corresponds to configurations deeper and deeper into the potential valleys.

The D-particles thus define a new perspective on the models introduced in section 1 . Their dynamics can be studied in these models and we refer to 29,30 for work along these lines. We should also mention that, depending on the kind of branes one is dealing with, other variants of supersymmetric quantum mechanics may be relevant. These can be obtained from the zerovolume limit of supersymmetric gauge theories coupled to matter. For instance, one such model was used in [30] to study the interaction between a 0-brane and a 4-brane. The two-dimensional model of section 4 may be regarded as a truncation of this nine-dimensional system.

From the quantum mechanics based on a finite number of degrees of freedom one may again hope to learn about the properties of D-branes. Some of these properties are again gouverned by duality arguments, in this case related to M-theory. This theory is conjectured to be the unified theory that encompasses all string theories (for a recent review, see [31]). It is eleven-dimensional in origin, but its structure is still largely unknown. String theories themselves do not live in eleven dimensions and should be viewed as perturbative realizations of M-theory. The small coupling is thus tied to the small size of certain compactified dimensions. At large distances M-theory should obviously be described by eleven-dimensional supergravity. When the size of the compactification is shrunk to zero, one describes the massless excitations of the corresponding string theory. The simplest such compactification, namely where one dimension is compactified on a circle, leads to IIA string theory. The size of the compactified coordinate, denoted by $R_{11}$ is identified as the ratio of the string coupling constant $g_{\mathrm{s}}$ and the string mass scale $M_{\mathrm{s}}$. By the standard Kaluza-Klein arguments, $R_{11}$ is also related to the Planck masses in eleven and ten dimensions, so that we obtain

$R_{11}=\frac{g_{\mathrm{s}}}{M_{\mathrm{s}}}=\frac{\left[M_{10}^{\text {Planck }}\right]^{8}}{\left[M_{11}^{\text {Planck }}\right]^{9}}$.

Furthermore we have the familiar ten-dimensional string relation between the Planck constant and the string scale, $M_{\mathrm{s}}^{8}=g_{\mathrm{s}}^{2}\left[M_{10}^{\text {Planck }}\right]^{8}$. Combining these equations leads to $M_{\mathrm{s}}=g^{1 / 3} M_{11}^{\text {Planck }}$ as well as to $R_{11}=g_{\mathrm{s}}^{2 / 3} / M_{11}^{\text {Planck }}$.

By various compactifications one can obtain the different types of string theories. In this way, the existence of M-theory explains the stringstring dualities that have been discovered in the last few years. These dualities were found both in the effective low-energy field theories based on different string vacua and by including solitonic $p$-branes into the various string theories and comparing their spectra (see e.g. [33,34).

The D-particles of IIA string theory can now be regarded as Kaluza-Klein states emerging in the compactification of eleven-dimensional super- 
gravity on $S^{1}$. The Kaluza-Klein states carry charges which are multiples of $1 / R_{11}$ and the Kaluza-Klein photon couples to them. However, viewed from a string perspective, the KaluzaKlein photon corresponds to one of the RamondRamond fields of IIA string theory, so that the massive Kaluza-Klein states carry RamondRamond charge. Consequently some of them can be identified with the D-particles. On the other hand, the IIA supergravity theory itself has solitonic solutions, extremal black holes, which are charged with respect to the Ramond-Ramond photon [32]. These particles are BPS states and leave only half the ten-dimensional supersymmetries invariant, precisely the same number as is left invariant by the massive Kaluza-Klein states! Hence they can be identified as D-particles [33].

Just as for the BPS monopoles, discussed earlier, one expects bound states for D-particles, precisely at threshold. Their existence can be inferred from the Kaluza-Klein spectrum of elevendimensional supergravity. In this way one may be able to verify some of the implications of duality with M-theory in the context of the models introduced in section 1. However, as we discussed in the previous section, to prove or disprove the existence of normalizable states within the continuum, is still a formidable task.

In summary, the situation is quite intriguing. On the one hand, supermembranes are just models in quantum mechanics associated with $U(N)$ in the large- $N$ limit. These supermembranes can live in a background of eleven-dimensional supergravity, which in itself is a very nontrivial property, but they are not viable as an elementary supermembrane in view of their continuous mass spectrum. On the other hand, the elevendimensional membrane provides, upon suitable compactification and in certain truncations, the various string excitations, including the solitonic ones. At the same time, the $N \rightarrow \infty$ quantum mechanics that describes the supermembrane, can be regarded as a theory of infinitely many D-particles. In a Kaluza-Klein perspective, these are just the massive Kaluza-Klein states of elevendimensional supergravity, which, when combined with the ten-dimensional IIA supergravity states, constitute eleven-dimensional supergravity com- pactified on a circle of finite radius. Apparently we are dealing with many faces of a single theory. In fact, one could turn the argument around and argue that it is the quantum-mechanical models that are fundamental. Recently this has led to the conjecture that the $N \rightarrow \infty$ limit is in fact a representation of uncompactified M-theory [35]. Irrespective of whether this is the right approach or not, these and related questions will eventually be clarified in the context of the elusive M-theory.

\section{Acknowledgements}

A substantial part of this work is based on results reported in [3, 0, 11, 12]. The work is supported by the European Commission TMR programme ERBFMRX-CT96-0045. I am grateful to H. Nicolai and E. Rabinovici for valuable discussions.

\section{REFERENCES}

1. M. Claudson and M.B. Halpern, Nucl. Phys. B250 (1985) 689; R. Flume, Ann. Phys. 164 (1985) 189; M. Baake, P. Reinicke, and V. Rittenberg, J. Math. Phys. 26. (1985) 1070.

2. M. Lüscher, Nucl. Phys. B219 (1983) 233; B. Simon, Ann. Phys. 146 (1983) 209.

3. B. de Wit, M. Lüscher and H. Nicolai, Nucl. Phys. B320 (1989) 135.

4. E. Bergshoeff, E. Sezgin and P.K. Townsend, Phys. Lett. 189B (1987) 75.

5. E. Bergshoeff, E. Sezgin and P.K. Townsend, Ann. Phys. 185 (1988) 330; P.K. Townsend, in "Superstrings '88", proc. of the Trieste Spring School, eds. M.B. Green, M.T. Grisaru, R. Iengo and A. Strominger (World Scient., 1989); M.J. Duff, Class. Quantum Grav. 5 (1988) 189.

6. A. Achúcarro, J.M. Evans, P.K. Townsend and D. L. Wiltshire, Phys. Lett. 198B (1987) 441.

7. B. de Wit, J. Hoppe and H. Nicolai, Nucl. Phys. B305 [FS23] (1988) 545.

8. J. Goldstone, unpublished.

9. J. Hoppe, in proc. Int. Workshop on Constraint's Theory and Relativistic Dynamics, eds. G. Longhi and L. Lusanna (World Sci- 
ent., 1987).

10. E.A. Bergshoeff, E. Sezgin and Y. Tanii, Nucl. Phys. B298 (1988) 187.

11. B. de Wit, U. Marquard and H. Nicolai, Commun. Math. Phys. 128 (1990) 39.

12. B. de Wit and H. Nicolai, in proc. Trieste Conference on Supermembranes and Physics in $2+1$ dimensions, p. 196, eds. M.J. Duff, C.N. Pope and E. Sezgin (World Scient., 1990).

13. E. Witten, Nucl. Phys. B188 (1981) 513.

14. A.V. Smilga, Yad. Fiz. 42(1985) 728; 43 (1986) 215; Nucl. Phys. B266 (1986) 45.

15. C.N. Pope and K.S. Stelle, Class. Quantum Grav. 5 (1988) L161.

16. A.J. Niemi and L.C.R. Wijewardhana, Phys. Lett. 138B (1984) 389; R. Blanckenbeckler and D. Boyanosvsky, Phys. Rev D30 (1984) 1821; D31 (1985)2089; D. Bollé, F. Gesztesy, H. Grosse, W. Schweiger and B. Simon, J. Math. Phys. 28 (1987) 1512.

17. J. Fröhlich and J. Hoppe, On zero-mass ground states in super-membrane matrix models, hep-th/9701119.

18. A. Kubek, Diplomarbeit (unpublished).

19. J. Harvey, Magnetic Monopoles, Duality and Supersymmetry, hep-th/9603086.

20. M. Atiyah and N. Hitchin, Phys. Lett 107A (1985) 21; The Geometry and Dynamics of Magnetic Monopoles, Princeton Univ. Press, 1988.

21. J.P. Gauntlett, Nucl. Phys. B400 (1993) 103, B411 (1994) 443; J. Blum, Phys. Lett. B333 (1994) 92.

22. E. Witten, Nucl. Phys. B202 (1982) 253.

23. A. Sen, Phys. Lett. B329 (1994) 217.

24. G.W. Gibbons and N.S. Manton, Nucl. Phys. B274 (1986) 183, Phys. Lett. B356 (1995) 32.

25. M. Porrati, Phys. Lett. B377 (1996) 76.

26. J. Polchinski, S. Chaudhuri and C.V. Johnson, Notes on D-branes, hep-th/9602052; J. Polchinski, TASI Lectures on D-branes, hepth/9611050: C. Bachas, (Half) A lecture on $D$-branes, lectures given at the Workshop on Gauge Theories, Applied Supersymmetry and Quantum Gravity, London, 1996 (hepth/9701019).

27. J. Polchinski, Phys. Rev. Lett. 75 (1995) 4724 (hep-th/9510017).

28. E. Witten, Nucl. Phys. B460 (1996) 335 (hepth/9510135).

29. U. Danielson, G. Ferretti and B. Sundborg, Int. J. Mod. Phys. A11 (1996) 5463 (hepth/9603081); D. Kabat and P. Pouliot, Phys. Rev. Lett. 77 (1996) 1004 (hep-th/9603127).

30. M.R. Douglas, D. Kabat, P. Pouliot and S.H. Shenker, D-branes and short distances in string theory, hep-th/9608024.

31. P.K. Townsend, Four lectures on M-theory, lectures given at the 1996 ICTP Summer School in High Energy Physics and Cosmology, Trieste (hep-th/9612121).

32. G. Horowitz and A. Strominger, Nucl. Phys. B360 (1991) 197.

33. P.K. Townsend, Phys. Lett. B350 (1995) 184 (hep-th/9501068), Phys. Lett. B373 (1996) 184 (hep-th/9512062).

34. E. Witten, Nucl. Phys. B443 (1995) 85 (hepth/9503124).

35. T. Banks, W. Fischler, S.H. Shenker and L. Susskind, M-theory as a matrix model: a conjecture, hep-th/9610043. 